\title{
Novel Nanotrees of Crystalline Nickel Formed via Electrolytic Approach
}

\author{
Jian Wang, Liying Zhang*, Liangming Wei, Chuanhai Jiang, Yafei Zhang*
}

((Received 10 December 2011; accepted 20 December; published online 30 December 2011.) )

\begin{abstract}
Tree-shaped nickel nanocrystals with long main trunk and highly ordered branches were prepared via electrolytic method in ethylene glycol solution. The morphology and structures of nanotrees of crystalline nickel can be controlled by the processing of the surfactant (PVP) and electrolytic voltage. Magnetic property measurements at room temperature indicated that the as-prepared nanotrees of crystalline nickel possess higher saturation magnetism $\left(M_{S}\right)$ and coercivity $\left(H_{C}\right)$ than those of corresponding bulk nickel materials.
\end{abstract}

Keywords: Nickel crystals; Nanotrees; Hysteresis loop; Thermogravimetric analysis

Citation: Jian Wang, Liying Zhang, Liangming Wei, Chuanhai Jiang and Yafei Zhang, "Novel Nanotrees of Crystalline Nickel Formed via Electrolytic Approach", Nano-Micro Lett. 3 (4), 264-269 (2011). http://dx. doi.org/10.3786/nml.v3i4.p264-269

\section{Introduction}

Nanoscale metal materials with different dimensions and shapes have attracted intensive interest due to their excellent electronic, magnetic, catalytic, optical properties and promising applications in many fields such as high-density magnetic recording, catalysis, biological labeling, optoelectronics, surface-enhanced Raman scattering (SERS), and so on [1-5]. During the past several years, various morphologies of nanoscale nickel $(\mathrm{Ni})$ such as nanoparticles, nanoplates, hexagonal nanodisks, nanotubes, nanowires, hollow octahedrons, concave polyhedrons, nanowalls and nanostrips, have indeed been synthesized [6-10]. Recently, one of the most significant breakthroughs in this domain was the preparation of the hierarchical structure [11,12]. Thanks to intensive efforts in nanomaterials for synthesizing the branched structures with well controlled sizes, shapes, and unique structures, some new opportunities have been offered with investigating the influence of morphologies and structures with respect to optical, electronic, magnetic and thermal properties of the branched nanocrystals [13-18]. In other words, all these unique properties critically depend on the controlled synthesis of branched nanocrystals, therefore, how to selective synthesizing unique metal nanomaterials with dendritic structures have not only fundamental scientific but also practical significance.

Although there have been many published reports pertaining to the synthesis of various dendritic crystals in the prior research, it is still important to develop simple and novel synthetic strategies that enable control over the products dimensions and shapes. For instance, Liu et al. have prepared Ni dendritic crystals with lengths about $0.5-2 \mu \mathrm{m}$ and thicknesses up to 10$30 \mathrm{~nm}$ via a facile hydrothermal reducing reaction of nickel dichloride in the presence of structure-directing agent (cetyltrimethylammonium bromide) [19]. Zhou et al. have also synthesized single crystalline $\mathrm{FeNi}_{3}$ dendrites with total lengths about $1-5 \mu \mathrm{m}$ by directly reducing the $\mathrm{Fe}(\mathrm{III})$ and $\mathrm{Ni}(\mathrm{II})$ salts with hydrazine hydrate in alkaline solution [20]. However, to the best of our knowledge, there have only a few reports about the large-scale synthesis of nanotree of crystalline $\mathrm{Ni}$ by the electrolytic method. In the present work, our research group demonstrates the electrolytic procedure that al-

Key Laboratory for Thin Film and Microfabrication of the Ministry of Education, Research Institute of Micro / Nano Science and Technology, School of Materials Science and Engineering, Shanghai Jiao Tong University, Shanghai 200240, China

*Corresponding authors. E-mail: liyingzhang@sjtu.edu.cn, yfzhang@sjtu.edu.cn 
low for the production nanotrees of crystalline Ni which have a hierarchical structure with primary, secondary, tertiary, and even higher-order branches.

\section{Experimental section}

\section{Preparation of the nanotrees of crystalline $\mathrm{Ni}$}

All chemicals used in our experiments were of analytical reagent grade and were directly used without further purification. In a typical procedure, $2.38 \mathrm{~g}$ of nickel chloride hexahydrate $\left(\mathrm{NiCl}_{2} \cdot 6 \mathrm{H}_{2} \mathrm{O}\right.$, Sinopharm Chemical Reagent Co., Ltd, China) was heated at $170^{\circ} \mathrm{C}$ for $60 \mathrm{~min}$ in a heat treatment furnace in open air, which entirely lost the crystal water and became the yellowbrown anhydrous nickel chloride $\left(\mathrm{NiCl}_{2}\right)$. Then $\mathrm{NiCl}_{2}$ was dissolved into $200 \mathrm{ml}$ ethylene glycol (EG, Shanghai Chemical Reagents Company, China) under continuous magnetic stirring at room temperature. After about 10 min, $30 \mathrm{ml}$ ethylene glycol solution of ploy(vinyl pyrrolidone) (PVP, $1.5 \mathrm{~g}, \mathrm{Mw} \approx 40000$, Sinopharm Chemical Reagent Co., Ltd, China) were added dropwise into the above ethylene glycol containing $\mathrm{Ni}^{2+}$ ions. Half an hour continuous magnetic stirring was carried out to ensure that $\mathrm{Ni}^{2+}$ ions and PVP were dispersed homogeneously in the solution to obtain a homogeneous grass-green solution, and subsequently transferred into electrolytic cell. The specific electrolytic process was carried out in a homemade two-electrode cell in the EG solution containing nickel chloride and two graphite plates as the anode and cathode, respectively. The distance between the two electrodes was about 300 $\mathrm{mm}$, and the graphite plate had a rectangular size of $500 \mathrm{~mm} \times 500 \mathrm{~mm} \times 10 \mathrm{~mm}$. Besides, the applied voltage was provided and controlled with the constant voltage mode by a direct-current (DC) voltage-stabilized power supply $(20-100 \mathrm{~V})$. After about $5 \mathrm{~h}$, the reaction was completed the grey-black fluffy solid were adsorbed on the surface of the cathode (graphite plates). The products were separated from the solution using magnetic field and washed three times with distilled water and ethanol, respectively, and then the products were dried in a vacuum oven at $60^{\circ} \mathrm{C}$ for $12 \mathrm{~h}$. Another simple was prepared using a similar procedure but without the introduction of ploy(vinyl pyrrolidone) (PVP) in the complex.

\section{Characterization}

The crystalline phases of the as-prepared products were identified by X-ray powder diffraction (XRD) using a advanced X-ray diffractometer (D8 ADVANCE, Bruker, Germany) in a 2 theta range from 35 to 90 degree with $\mathrm{Cu} \mathrm{K} \alpha(\lambda=0.154056 \mathrm{~nm})$ rotating anode point source operating at $40 \mathrm{kV}$ and $40 \mathrm{~mA}$. The size and surface morphology measurements were performed by using an emission scanning electron microscopy (SEM,
Zeiss Ultra 55, Germany) at an accelerating voltage of $5 \mathrm{kV}$. SEM samples were diluted 20 times with acetone and then deposited on silicon substrates. Thermogravimetric analyses of the samples prepared were carried out on a thermogravimetric analyzer (TGA, Pyris 1, USA) with a rising temperature rate $10^{\circ} \mathrm{C} / \mathrm{min}$ in flowing air. The magnetic hysteresis loops of the samples were carried out at $300 \mathrm{~K}$ in a vibrating sample magnetometer (VSM, Lake Shore 7400, USA) in a magnetic field of up to 10000 Oe. For magnetization measurements, the powders were pressed strongly and fixed in a small cylindrical plastic box.

\section{Results and discussion}

The XRD patterns of the products prepared with and without the introduction of ploy(vinyl pyrrolidone) (PVP) are similar. Figure 1(A) shows the typical XRD patterns of the as-prepared samples with and without PVP introduced. All the diffraction peaks of these two samples match well with the nickel face-centered cubic (FCC) structure (PDF standard cards, Joint Committee on Powder Diffraction Standards (JCPDS) card No. 04-0850). These peaks at the scattering angles $(2 \theta)$ of $44.70^{\circ}, 52.00^{\circ}$ and $76.70^{\circ}$ correspond to crystal planes of (111), (200) and (220) of crystalline nickel, respectively. From the XRD patterns, no characteristic peaks of impurities, such as nickel oxide phase or nickel hydroxide phase, were detected, indicating that pure nickel phase was obtained using this one-step, electrolytic route with or without PVP introduced at atmospheric pressure. The average crystalline grain size of the sample was calculated from the XRD patterns according to the Scherrer formula:

$$
D=\frac{k \cdot \lambda}{\beta \cdot \cos \theta}
$$

where $\mathrm{D}$ is the average crystalline grain size, $k$ is the Scherrer constant related to the shape and index (hkl) of the crystals, $\lambda$ is the wavelength $(0.154056 \mathrm{~nm})$ of the $\mathrm{X}$-Ray, $\beta$ is the FWHM (full width at half maximun), and $\theta$ is the diffraction angle. The average crystalline grain size of the as-synthesized nanotrees of crystalline $\mathrm{Ni}$ was estimated about $20 \mathrm{~nm}$, which was obviously smaller than the diameter of the sample in SEM images (Fig. 1(B2) or (C1)). This result illustrates that the nanotree structures was assembled by lots of crystalline grains during the electrolytic process.

Figure 1(B1) presents the low-magnification scanning electron microscopy (SEM) image of the as-prepared nanotrees of crystalline $\mathrm{Ni}$ via the electrolytic process with $1.5 \mathrm{~g}$ ploy(vinyl pyrrolidone) (PVP) introduced. The morphology of the sample has been identified as uniform nanotree structures which consists almost entirely of such hierarchical structure sprawling to several 

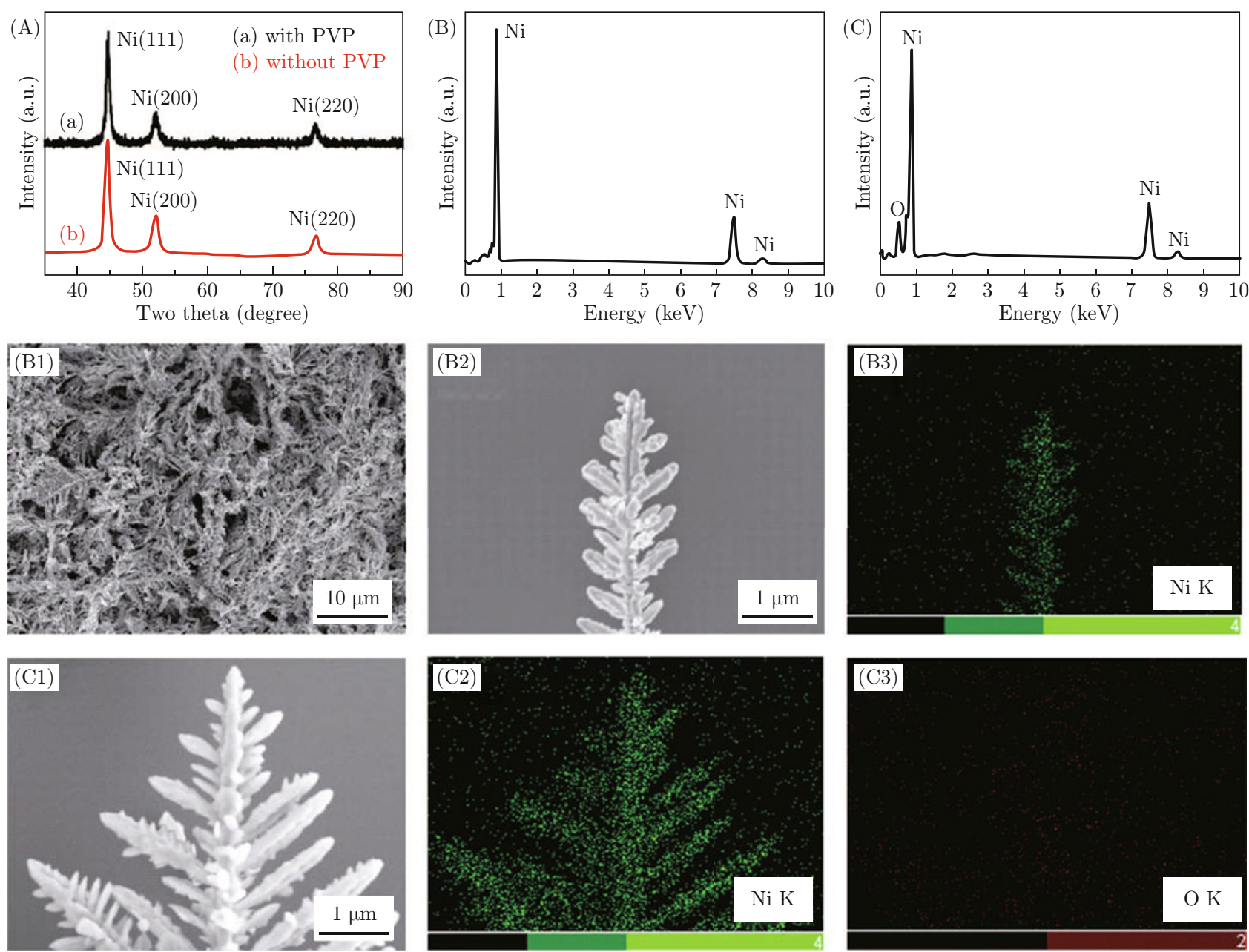

Fig. 1 (A) XRD patterns of the nanotrees of crystalline Ni prepared with (a) and without (b) 1.5 g ploy(vinyl pyrrolidone) (PVP) introduced. Energy-dispersive X-ray spectrum (EDS) images of the Ni nanotree crystals prepared with (B) and without (C) PVP introduced. (B1), (B2) and (B3) show SEM images and the corresponding EDS the elemental distribution of $\mathrm{Ni}$ in nanotrees prepared with PVP introduced. (C1), (C2) and (C3) show SEM image and the corresponding EDS mapping of the elemental distribution of $\mathrm{Ni}$, and $\mathrm{O}$ in nanotrees prepared without PVP introduced.

generations. The high-magnification image in Fig. 1(B2) clearly demonstrates the morphology of a fractional dendrite, which reveal a well-defined nanotree structure with a long main trunk and highly ordered short side branches distributed on both sides of the trunk. Figure 1(C1) clearly shows a fractional nanotree structure prepared without PVP introduced during the electrolytic process. In order to better understand well about the surface element of the nanotrees of crystalline $\mathrm{Ni}$, further Energy-dispersive X-ray spectrum (EDS) analysis (Fig. 1(B) with PVP introduced, and Fig. 1(C) without PVP introduced) revealed that the nanotrees of crystalline $\mathrm{Ni}$ prepared with PVP introduced during the electrolytic and collective process were not oxidized, because PVP prevented the oxygen entering nanotree structures. The amorphous nickel oxide layer on the surface of the Ni nanotrees prepared without PVP introduced has been confirmed by using
EDS mapping mode (see Fig. 1(B3), (C2) and (C3)). The weak $\mathrm{O}$ signal across the nanotree structure confirms the amorphous nickel oxide (see Fig. 1(C3)), but not crystalline nickel oxide (see Fig. 1(A)).

To determine the stability of the Ni nanotree, thermogravimetric analyzer (TGA) measurements were performed on the purified samples of $\mathrm{Ni}$ nanotrees under air flow. As shown in Fig. 2(a), the pure nanotrees of crystalline $\mathrm{Ni}$ began to be oxidized around $375^{\circ} \mathrm{C}$, the final weight gain was about $23 \%$, which was close with the theoretical weight gain value of $21.3 \%$ calculated for the change of pure nickel to nickel oxide crystals. This thermogravimetric result indicates that the sample consists of pure nickel, and the oxidation process from nickel to nickel oxide is complete yet at about $800^{\circ} \mathrm{C}$. Figure $2(\mathrm{~b})$ shows a weight decline pattern with the inflexion points at about $400^{\circ} \mathrm{C}$. This change corresponds to the degradation of surfactant (PVP) 


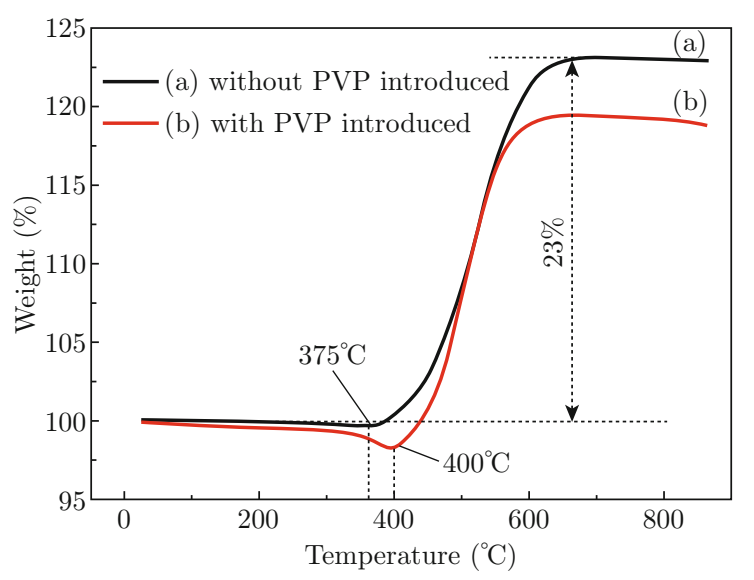

Fig. 2 Thermogravimetric analysis of the nanotrees of crystalline $\mathrm{Ni}$ prepared without (a) and with (b) $1.5 \mathrm{~g}$ PVP introduced.

molecules that probably formed a sheath around each Ni nanotree [21].

The magnetic properties of two nickel nanostructured samples have been measured by VSM. As shown in Fig. 3(a), the coercivity $\left(H_{C}\right)$, saturation magnetization $\left(M_{S}\right)$, and remnant magnetization $\left(M_{r}\right)$ of the crystalline $\mathrm{Ni}$ nanotrees for the samples without PVP introduced are $189.52 \mathrm{Oe}, 48.52 \mathrm{emu} / \mathrm{g}$ and $14.26 \mathrm{emu} / \mathrm{g}$, respectively. However, for the samples with $1.5 \mathrm{~g}$ PVP introduced, the values are respective $232.34 \mathrm{Oe}, 41.95 \mathrm{emu} / \mathrm{g}$ and $12.44 \mathrm{emu} / \mathrm{g}$ (see Fig. 3(b). It can be found that the $H_{C}$ value for the sample with PVP is larger than that for without PVP. This can be easily understood that there formed more preferred magnetic orientation structures in the sample with PVP, that is, the introduction of PVP is conductive to obtain multi-dendrites Ni nanostructures in this approach, which consists with the above SEM results. However, the $M_{S}$ value for the sample with PVP is less than that for without PVP due to the existant of non-magnetic PVP in the sample. It also can be found that the $H_{C}$ and $M_{r}$ values for these two sample are much higher than those for bulk nickel materials (100 Oe, $2.7 \mathrm{emu} / \mathrm{g}$ ), which also can be attributed to their unique preferred orientation structures [22] and the reduced size as well as presence of hierarchical structures [7]. The less $M_{S}$ for these two samples compared with that for bulk nickel materials ( $55 \mathrm{emu} / \mathrm{g}$ ) is mainly due to the spin disorder on the surface and surface oxidation [2]. The M-H hysteresis loops of two nickel samples are all symmetric in shape with respect to zero magnetic field. This demonstrates that there is no exchange biasing effect which is usually induced by the presence of nickel oxide $[7,23]$.

Nanotrees of crystalline Ni were synthesized via electrolytic process, as shown in Fig. 4(a), (b), (c), and (d). In a typical experiment, $\mathrm{Ni}^{2+}$ ions with positive charges moved towards the cathode, $\mathrm{Cl}^{-}$ions simultaneously moved towards the anode under the electric field. The specific electrolytic process can be expressed as follows [24]:

$$
\begin{aligned}
& \text { Cathode }: \mathrm{Ni}^{2+}+2 \mathrm{e}^{-} \rightarrow \mathrm{Ni} \\
& \text { Anode }: 2 \mathrm{Cl}^{-}-2 \mathrm{e}^{-} \rightarrow \mathrm{Cl}_{2} \uparrow \\
& \text { Overallreaction : } \mathrm{NiCl}_{2} \rightarrow \mathrm{Ni}+\mathrm{Cl}_{2} \uparrow
\end{aligned}
$$

We find that the surfactant (PVP) is of great importance for controlling the structure and morphology of Ni nanotrees. Figure 4(A) and (B) show the single Ni nanotree prepared with and without $1.5 \mathrm{~g}$ PVP introduced at $45 \mathrm{~V}$, respectively, and the corresponding schematic is shown in Fig. 4(a) and (b). When the surfactant (PVP) is used during electrolytic process, more multi-dendrites were formed and orderly assembled. This multi-nanotree structure consists of several
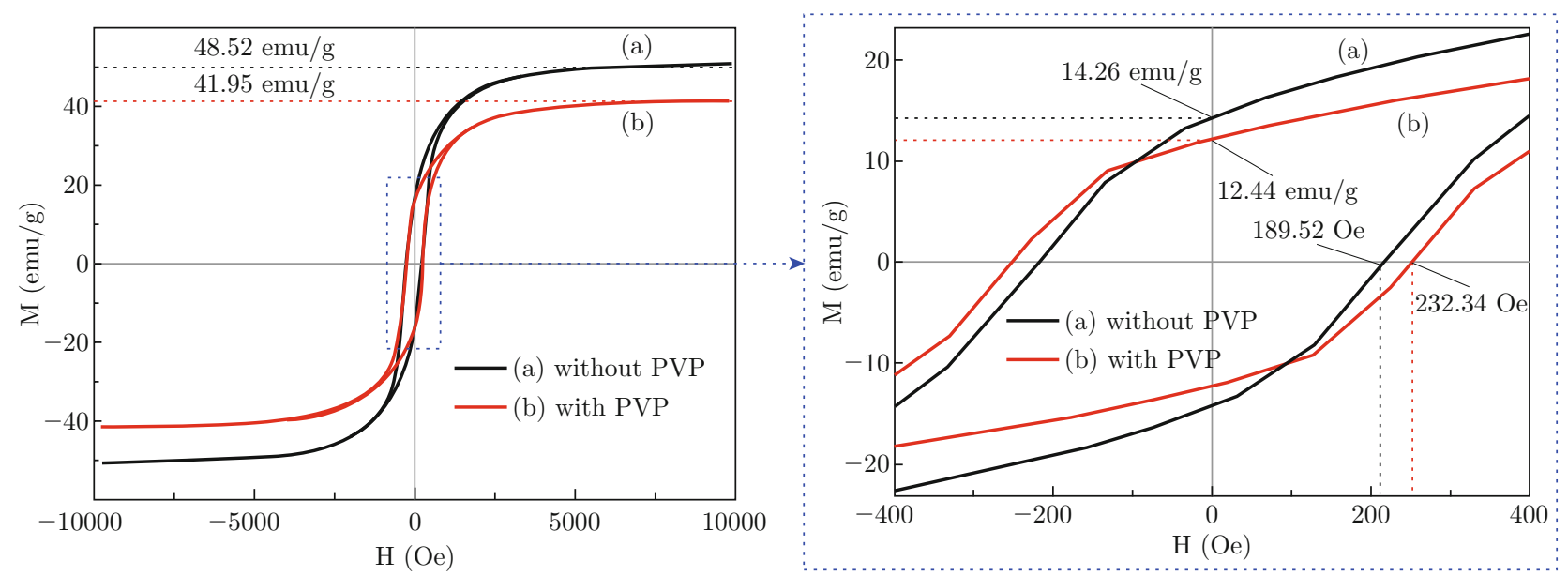

Fig. 3 M-H hysteresis loops (at $300 \mathrm{~K}$ ) of the Ni nanotrees synthesized without (a) and with (b) $1.5 \mathrm{~g}$ PVP introduced. 

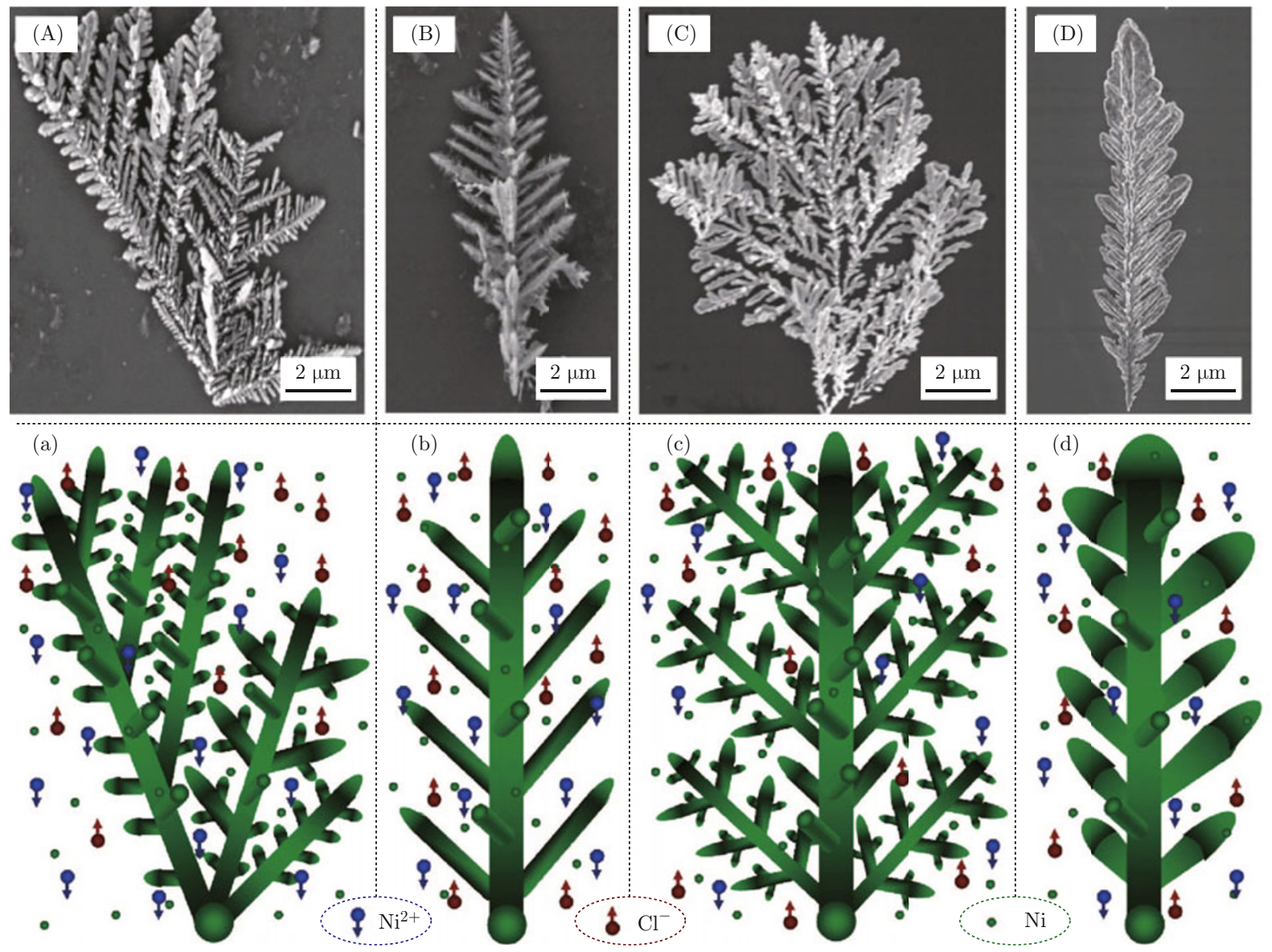

Fig. 4 SEM images and the corresponding schematic of the single Ni nanotree prepared with ((A), (a)) and without ((B), (b)) $1.5 \mathrm{~g}$ PVP introduced via electrolytic processes. SEM images and the corresponding schematic of the single Ni nanotree prepared at higher voltage of $120 \mathrm{~V}((\mathrm{C}),(\mathrm{c}))$ and lower voltage of $30 \mathrm{~V}((\mathrm{D}),(\mathrm{d}))$ during electrolytic processes.

trunks and many abundant branches, and every branch is an assembly of rod-like nanoclusters, as shown in Fig. 4(A). However, when PVP was not introduced, (see Fig. 4(B)), the Ni nanostructure is featured by dendrites with single trunk and symmetrical branches, and the trunk are approximately $15 \mu \mathrm{m}$ in length. Moreover, the electrolytic voltage for synthesis of Ni nanotrees is also an important factor which affects the metal crystal growth rate, and also the morphology and structure of the products. Figure $4(\mathrm{C})$ and (D) show the single Ni nanotree prepared without PVP introduced via electrolytic process at higher voltage (120 $\mathrm{V})$ and lower voltage $(30 \mathrm{~V})$, respectively, and the corresponding schematics are shown in Fig. 4(c) and (d). At a higher electrolytic voltage of $120 \mathrm{~V}$, the morphology of the product was multi-dendrites composed of a trunk and much more secondary and tertiary higherorder branches. When the electrolytic voltage was decreased to $30 \mathrm{~V}$, the trunks and branches of the nanotree structures become thick and short, and the tip of the trunk and branches consist of the nanoplates and microspheres.

\section{Conclusions}

In summary, we demonstrated a practical, electrolytic approach to the large-scale synthesis of magnetic $\mathrm{Ni}$ nanotrees which revealed a well-defined nanotree structure with a long main trunk and highly ordered short side branches distributed on both sides of the trunk. We found that the surfactant and electrolytic voltage would significantly influence the morphology and structure of the Ni nanotrees. From the TGA characterization, we also observed that Ni nanotrees are stable in air when the temperate is below $375^{\circ} \mathrm{C}$. The as-prepared Ni nanotrees possess higher $\mathrm{M}_{\mathrm{S}}$ and $\mathrm{H}_{\mathrm{C}}$ than those for corresponding bulk materials.

It should be suggested suggest that the approach we demonstrated here could also be extended to fabricate other magnetic metal nanotree structures.

\section{Acknowledgement}

We thank the generous financial support from Natural Science Foundation of China (No. 50730008) and 
shanghai (No. 10ZR1416300), Shanghai Science and Technology Grant (No. 1052nm05500), and the Foundation for SMC Excellent Young Teacher in Shanghai Jiao Tong University.

\section{References}

[1] Y. G. Sun and Y. N. Xia, Science 13, 298 (2002).

[2] H. Niu, Q. Chen, M. Ning, Y. Jia and X. Wang, J. Phys. Chem. B 108, 3996 (2004). http://dx.doi.org/ 10.1021/jp0361172

[3] Y. W. Jun, Y. Y. Jung and J. Cheon, J. Am. Chem. Soc. 124, 615 (2002). http://dx.doi.org/10.1021/ ja016887w

[4] B. Kim, S. L. Tripp and A. Wei, J. Am. Chem. Soc. 123, 7955 (2001). http://dx.doi.org/10.1021/ ja0160344

[5] Y. H. Ni, X. W. Ge, Z. C. Zhang and Q. Ye, Chem. Mater. 14, 1048 (2002). http://dx.doi.org/10.1021/ $\mathrm{cm} 010446 \mathrm{u}$

[6] Ö. Metin, V. Mazumder, S. Özkar and S. Sun, J. Am. Chem. Soc. 132, 1468 (2010). http://dx.doi.org/10. 1021/ja909243z

[7] F. Jia, L. Zhang, X. Shang and Y. Yang, Adv. Mater. 20, 1050 (2008). http://dx.doi.org/10.1002/adma. 200702159

[8] S. J. Park, S. Kim, S. Lee, Z. G. Khim, K. Char and T. H. Hyeon, J. Am. Chem. Soc. 122, 8581 (2000). http://dx.doi.org/10.1021/ja001628c

[9] D. Ciuculescu, F. Dumestre, M. Comesana-Hermo, B. Chaudret, M. Spasova, M. Farle and C. Amiens, Chem. Mater. 21, 3987 (2009). http://dx.doi.org/10.1021/ cm901349y

[10] L. Zhang, J. Wang, L. Wei, P. Liu, H. Wei and Y. Zhang, Nano-Micro Lette. 1, 49 (2009).

[11] X. Liu, R. Li, Y. Wang, G. Qiu, N. Zhang and X. Li, J. Phys. Chem. C 111, 163 (2007). http://dx.doi.org/ 10.1021/jp0643597

[12] J. Fang, H. You, P. Kong, Y. Yi, X. Song and B. Ding, Cryst. Growth Des. 7, 864 (2007). http://dx. doi.org/10.1021/cg0604879
[13] M. H. Cao, T. F. Liu, S. Gao, G. B. Sun, X. L. Wu, C. W. Hu and Z. L. Wang, Angew. Chem. Int. Ed. 44, 4197 (2005). http://dx.doi.org/10.1002/anie. 200500448

[14] V. L. Colvin, M. C. Schlamp and A. P. Alivisatos, Nature 370, 354 (1994). http://dx . doi .org/10.1038/ $370354 \mathrm{a0}$

[15] R. P. Andres, J. D. Bielefeld, J. I. Henderson, D. B. Janes, V. R. Kolagunta, C. P. Kubiak, W. J. Mahoney and R. J. Osifchin, Science 273, 1690 (1996). http:// $\mathrm{dx}$.doi.org/10.1126/science.273.5282.1690

[16] A. Sukhanova, A. V. Baranov, T. S. Perova, J. H. M. Cohen, I. Nabiev, Angew. Chem. Int. Ed. 45, 2048 (2006). http://dx.doi.org/10.1002/anie. 200503016

[17] S. O. Cho, E. J. Lee, H. M. Lee, J. G. Kim, Y. J. Kim, Adv. Mater. 18, 60 (2006). http://dx.doi.org/ 10.1002/adma. 200501600

[18] G. Schmid, Chem. Rev. 92, 1709 (1992). http://dx. doi.org/10.1021/cr00016a002

[19] X. M. Liu and S. Y. Fu, J. Cryst. Growth 306, 428 (2007). http://dx.doi.org/10.1016/j. jcrysgro.2007.05.031

[20] L. P. Zhu, H. M. Xiao, W. D. Zhang, Y. Yang and S. Y. Fu, Cryst. Growth Des. 8, 1113 (2008). http://dx. doi.org/10.1021/cg701036k

[21] P. Liu, Z. Li, B. Zhao, B. Yadian and Y. Zhang, Mater. Lett. 63, 1650 (2009). http://dx.doi.org/10.1016/ j.matlet. 2009.04.031

[22] P. Saravanan, T. A. Jose, P. J. Thomas and G. U. Kulkarni, Bull. Mater. Sci. 24, 515 (2001). http://dx. doi.org/10.1007/BF02706724

[23] C. Liu, L. Guo, R. Wang, Y. Deng, H. Xu and S. Yang, Chem. Commun. 2726 (2004). http://dx.doi. org/10.1039/b411311j

[24] J. Wang, L. Wei, L. Zhang, Y. Zhang and C. Jiang, Cryst. Eng. Comm. In press, (2012). http://dx.doi. org/10.1039/C1CE06066J 